Peter Simeon Swisher Vfinity Ilana Holmes

Excite Media Group LLC 5 Union Square West, 4th FI New York, NY 10003, USA

Tel: +1 2129418499 ext 207,

E-mail: iholmes@excitepr. com, www.excitepr.com

\title{
The managed web: A look at the impact of Web 2.0 on media asset management for the enterprise
}

\section{Peter Simeon Swisher}

is the product manager at VFinity, an innovative content management software company based in NYC. VFinity has developed and implemented the only web-native asset management system on the market today. As product manager, Swisher helps guide development, sales and marketing for VFinity's products and services. For more than a decade, he has helped lead the release of digital asset management, relationship management and other web-based technologies for companies such as Quark, Inc., Aris Global and Interactive Data Corporation. He has lectured and written on strategies and uses of commercial software for the print media, financial, pharmaceutical and broadcasting industries. Swisher has a Bachelor of Arts degree from the University at Albany, NY.

Keywords: Web 2.0, multimedia asset management (MAM), MAM 2.0, digital asset management (DAM), digital media, broadcast DAM

Abstract This paper examines the fundamental shift occurring within multimedia asset management and Digital Asset Management solutions, a shift that has been ignited by Web 2.0 and its core principles; community-driven adaptive technologies that converge media, metadata, users, and tools - all via the web. This paper explores how the social networks and technology, which are driving the Web 2.0 phenomena, are expanding user adoption at alarming rates, drastically increasing re-use of licensed and unlicensed assets, as well as emphasizing a need for greater and more scalable governance of intellectual property and business rules for managing user generated content. Journal of Digital Asset Management (2007) 3, 32-42. doi:10.1057/palgrave.dam.3650061

\section{INTRODUCTION TO MAM 2. 0}

Just within the past few years, user-driven technology models - exemplified by the social phenomena known as "Web 2.0" - have created a fundamental shift in multimedia asset management (MAM) and how end users interact with it. MAM is being forced to evolve from closed client/server (C/S) enterprise models to increasingly open, metadata-centric and webnative architectures. This new model connects media, metadata, end users and production tools via the web in completely networked and userdriven ways. This is the "managed web", or "MAM 2.0".

Systems that exemplify Web 2.0, such as Wikipedia, Flickr, Amazon, eBay and YouTube are among the largest digital file management solutions in use today; in terms of users and assets under management it is hard to imagine larger digital asset management (DAM) communities. For example, as of August 2005 Flickr.com - a Web 2.0-based photo sharing and metadata tagging community - supported over 37,000,000 digital images and over 1,200,000 end users. ${ }^{1}$ Amazon has over $59,000,000$ customer accounts worldwide. Web 2.0 systems achieve these amazing usage levels within very small timeframes. For example, Flickr, as of 19 December 2006, had $324,000,000$ digital images and 6,000,000 registered users. ${ }^{2}$

Within the MAM 2.0 ecosystem, Context is King. ${ }^{3}$ Data such as how production tools and assets are used is logged and dynamically impacts the entire system. Feedback from consumers is immediate. Tracking and using context within MAM 2.0 drives further learning and selfmanagement for the entire ecosystem. The system becomes richer and more useful the more it is used.

MAM 2.0 removes boundaries between the community, media and metadata. Legacy MAM solutions created clear boundaries between content creators and content consumers. 
Content creators were within company walls; content consumers were at the receiving end of distribution packages. MAM 2.0 ecosystems blur these lines in such a way that consumers are also producers, and producers are directly connected to consumers via the same ecosystem.

At the same time, these user contributions means that proper re-use and management of protected materials is more important than ever before. This paper will explore the principles of MAM 2.0 and how it is fundamentally changing what users expect from MAM. Finally, it will investigate strategies for protecting licensed assets in a completely open and networked Web 2.0-ready MAM, MAM 2.0.

\section{WHY IS THIS DAM SYSTEM NECESSARY?}

Legacy DAM and MAM solutions offer significant value to the companies that choose to adopt them. Within existing networks, they improve all aspects of file management: including making ingesting, indexing, storage, retrieval, and re-use more efficient. Rights management and copy-protection also work extremely well inside of these closed environments. The explosion in the amount of digital media and the need to manage this media across any device or platform is, however, a great challenge even for the most well-established DAM companies.

\section{THE EMERGENCE OF THE SOCIALLY NETWORKED WEB}

In 1990, the first commercial internet service provider opened for business. ${ }^{4}$ By 1995, the number of internet hosts (a computer system with a registered internet protocol address) grew to $5,846,000 .{ }^{5}$ Still, in 1995 , the internet was available in only 14 per cent of US households. By 2000 that number had grown by 39 per cent. In 2000, the number of hosts had risen to $93,047,785$. As of July 2006 , that number was $439,286,364 .{ }^{6}$ As impressive, was the prevalence of the internet in the workplace. By 2003, over 87.5 per cent of full-time employees had internet access at work. ${ }^{7}$ The increased home and workplace usage of personal computers and the internet is causing a corresponding explosion in digital files and all-digital workflows.

Digital media has evolved to multimedia. Single-purpose files are now rich source files. Via MAM 2.0, multimedia source files may undergo multiple renditions to serve completely different distribution needs. The evolution of multimedia and the web and exponentially increasing user communities provides great promise and challenges for MAM. A new breed of solution is needed to meet these challenges. Blending the best of existing requirements and tools with emerging social networks fostered by Web 2.0 is the winning strategy.

From Web 1.0 to ...

For most of its existence, the web has functioned as a medium for publishing static information to passive audiences. The flow was simple: enterprises and institutions would publish information, and end users would consume it. This is also true for DAM solutions. Users created static collections of digital assets and administrators provided a view into those catalogs via a web front end to the MAM. Still, the presentation of information was static; it only changed when the institution decided it was time to publish new assets.

As the availability of the web in the office increased, DAM end users began requesting web interfaces to these solutions. Within the technical limitations of the time, vendors responded by offering functionally limited web front ends to $\mathrm{C} / \mathrm{S}$ systems. Many enterprise-scale DAM is still structured this way today. The most useful aspects of the DAM remain on the Client or Server level, while the web front end is used as a static presentation tool.

... to Web 2.0, the socially networked web

What has emerged within the past two to three years is a truly dynamic, user-driven and completely networked web. This "socially networked web" provides for live collaborations between the publisher and the audience. It is a web which improves the more it is used and the more open it is. It is a system which offers applications that are designed completely for the web; services such as Google documents and spreadsheets ${ }^{8}$ bring the power of Microsoft Word and Excel completely onto a web platform. Web 2.0 solutions not only manage more assets than 
any other DAM system, they do so in largely self-managed ways. To take advantage of this wonderful technological evolution, legacy DAM must adopt Web 2.0 principles, or be replaced with managed web solutions.

\section{WEB 2.0, MORE THAN JUST A BUZZWORD}

While Web 2.0 - like MAM before it - is in one sense flashy new technical jargon, what Web 2.0 represents is extremely important. Web 2.0 is the convergence via the web of an ecosystem of communities of users, business, tools and digital media, in such a way that the whole system becomes more useful and less difficult to manage the more it is used. It is a set of completely networked web applications and databases that are designed to adapt to the needs of its users, based upon how they actually use it, not on how vendors or employers want them to use it. Web 2.0 can be considered to be almost completely user-driven. This is in stark contrast to most MAM systems that become more difficult to manage the larger the asset and especially the user base grows.

\section{Web 2.0 patterns and principles of the managed web}

The core principles of MAM 2.0 and the core patterns of Web 2.0, as defined by O'Reilly Media, ${ }^{9}$ are listed below. More information and specific examples are provided throughout this paper. While there may be some overlap and confusion about the terms, this paper will address them as well (Figure 1).

\section{Metadata and media}

With workgroup and enterprise DAM, usage data such as how, when and where an asset was used is typically only available within annual usage reports. It is rarely used to affect any dynamic changes in system function, or to inform the choices of the user community. This is not the case with MAM 2.0. Whether data is implicitly extracted, or explicitly entered, any action within the enterprise MAM community - any file added, commentary, or metadata entered - is a contribution to the collective intelligence of the entire system. A great example of using transactional data to benefit the community is Amazon.com.

Data is the Next 'Intel Inside': Using unique, hardto-recreate data sources to become the 'Intel Inside' for this era in which data has become as important as function. ${ }^{10}$

Every transaction in Amazon adds to the global data pool, effectively altering the entire system.

A look at the profile page for the book

"Freakonomics" provides immediate access to the pooled transactional data about this product and the community that has purchased it. For example, we see that 31 per cent of the community who purchased "Freakonomics" also purchased "The Tipping Point" by Malcolm Gladwell. Another 31 per cent bought "The World is Flat" by Thomas L Friedman. Another 23 per cent paid for "Blink: The Power of Thinking Without Thinking".

Members of the Amazon community can immediately become part of numerous subcommunities based on what they purchase.

\begin{tabular}{|l|c|c|c|c|c|}
\hline \multicolumn{7}{|c|}{ C O R E P R I N C I P L E S O F M A M 2.0 } \\
\hline & MEDIA \& METADATA & TOOLS & $\begin{array}{c}\text { CONNECTING TOOLS } \\
\text { \& MEDIA }\end{array}$ & $\begin{array}{c}\text { SOCIAL } \\
\text { SOFTWARE }\end{array}$ & $\begin{array}{c}\text { RICH USER } \\
\text { EXPERIENCES }\end{array}$ \\
\hline Harnessing Collective Intelligence & & & & & \\
\hline Data Is the Next "Intel Inside" & & & & & \\
\hline Innovation in Assembly & & & & & \\
\hline Rich User Experiences & & & & & \\
\hline Software Above the Level of a Single Device & & & & & \\
\hline Perpetual Beta & & & & & \\
\hline Leveraging the Long Tail & & & & & \\
\hline Lightweight Models and Cost-Effective Scalability & & & & MAM.2.0 @2006 GISTICS \\
\hline
\end{tabular}

Figure 1: Matrix of Web 2.0 patterns and core principles of MAM 2.0 
They can more easily delve deeper into the topics that interest them, and will be informed by a community of like-minded users, via "social software". Instead of locking this communitygenerated data away until quarterly reporting time, Amazon made it intrinsic to the ecosystem. Given that Amazon has over 59,000,000 members, this was no small task.

The same richness is beneficial to MAM 2.0. How, when, where, why and to what effect corporate assets - such as logos, Adobe InDesign layouts or Final Cut Pro projects are used, is unique valuable data. MAM 2.0 should be able to leverage this information to help make better decisions based on the user community.

\section{Connect digital content with digital tools via the web}

Legacy DAM and organizational structures create many types of silos. One example of this is legacy DAM, MAM and rights management solutions cannot communicate with each other. Tool silos are created by incompatible devices and formats, or by devices that simply cannot work on the platforms and communication protocols that a global organization requires. Organizational silos evolve as well; editors are blocked from your producers, producers are blocked from contractors and so forth. Rights management systems are blocked from payment systems. Desktop asset management is blocked from another department's workgroup asset management system; the workgroup system is blocked from the enterprise system, which in turn is blocked from a large percentage of your corporation. And as is often the case with DAM, your customers are blocked from you.

Software Above the Level of a Single Device: Create software that spans Internet-connected devices and builds on the growing pervasiveness of online experience. $^{11}$

MAM 2.0 eliminates these silos by either replacing them or bridging them. Producers collaborate with contractors and customers via completely web-based tools, media and metadata. Editors and managers have immediate access to assets regardless of when they connect or what tool or operating system or location they are connecting from. Assets and metadata from one system or workflow flow seamlessly into other systems and workflows. Legacy devices and databases are not eliminated, they are leveraged. Open standards and web-based solutions help make this possible with MAM 2.0.

\section{Social software}

Social software is web-native technology that is available to entire communities and improves the more it is used. Like any legacy DAM, powerful and flexible metadata controls are required. In MAM 2.0 however, users may have open access to metadata. The more metadata they add, and the more valuable their metadata, the more useful the system becomes. Harnessing this "Collective Intelligence" is a fundamental tenet of Web 2.0.

Harnessing Collective Intelligence: Create an architecture of participation that uses network effects and algorithms to produce software that gets better the more people use it. ${ }^{12}$

Systems that use collective intelligence principles will make it very easy for users to contribute data, whether it is done implicitly or explicitly. The user's own interests will drive adoption. Also, data from the usage of integrated production tools should add to the collective intelligence. How a Final Cut Pro user works, what files they use, how they use them, when, and where is all valuable data. Tag Clouds are a popular example of what can be done with collective intelligence after it's been harnessed (Figure 2).

Tag clouds are a way to browse to assets via metadata assigned to that asset by the community. It is not unlike browsing via a keyword index or structured taxonomy. With MAM 2.0 however, the browsing experience changes every time the system is used. The more a metadata value is used, the more prominent it will be in the tag cloud. Tag clouds can be implemented in any number of ways, and are not limited to the example given below.

\section{Closed solutions cannot harness collective intelligence}

Collective intelligence cannot be harnessed if people do not participate or if the software cannot leverage their participation. Systems that offer closed or proprietary metadata schemas and 
06 africa amsterdam animals architecture art august australia autumn baby barcelona beach berlin birthday black blackandwhite blue boston bw california cameraphone camping canada canon car cat cats chicago china christmas church city clouds color concert day dc dog england europe fall family festival film florida flower flowers food france friends fun garden geotagged germany girl graffiti green halloween hawaii hiking holiday home honeymoon hongkong house india ireland island italy japan july june kids lake landscape light live london losangeles macro may me mexico mountain mountains museum music nature new newyork newyorkcity newzealand night nikon nyc ocean october paris park party people portrait red river roadtrip rock rome san Sanfrancisco school scotland sea seattle september show sky snow spain spring street summer sun sunset sydney taiwan texas thailand tokyo toronto travel tree trees trip uk urban usa vacation vancouver washington water wedding white winter yellow york zoo

Figure 2: Tag cloud from Flickr.com

principles, will struggle to adopt collective intelligence in any meaningful way. Likewise, if the software requires significant training and customization, then the benefits of collective intelligence will not be realizable.

\section{The smart kid is not always right}

Unleashing Collective intelligence presents significant issues for MAM 2.0 solution. It provides access to an unparalleled array of data. The quality of the media and metadata will directly impact the quality of the ecosystem within which it exists. The managed web must offer solutions to filtering and controlling this less valuable data.

In some cases, the community will be trusted to self-manage. Rating systems, notification and violation controls, and voting mechanisms are some ways that MAM 2.0 offers selfmanagement. Group level moderation is also possible; moderators keep the community on track, and remove abusive members as necessary. Whether self-managed, or moderated, or automanaged by rights management solutions, MAM 2.0 solution supports whatever model is most effective.
If the tail is long enough, then grab it Another foundational aspect of Web 2.0, "Leveraging the Long Tail" refers to exploiting to new uses and distribution points for all your content - even older, less popular content. Amazon.com for example, has made more money selling this type of content than they have from selling top 40 hits.

Leveraging the Long Tail: Capture niche markets profitably through low-cost economics and broad reach enabled by the Internet. ${ }^{13}$

Depending on your viewpoint, one potential example of long tail content is "Pocket Full of Kryptonite" from the Spin Doctors. This was once an extremely popular album. It was, however, played so often for a time-period in the early 1990s, that the public became oversaturated by it. The band also failed to produce new material. As a result, the album fell from favor. One would struggle to find a brick and mortar store that carries it today. Does this mean the album can never be sold again? No.

In the digital world, it does not matter how much shelf space a product takes up, because there is no shelf. Amazon makes this album 
available to users in a myriad number of ways. Fans can search for it explicitly, or they can find it indirectly; for example while viewing one of the band's major influencers. Brick and mortar business cannot do this because of limited and expensive shelf space. With sites like Amazon, passionate Spin Doctors fans can easily find a like-minded community where they can download their music and videos and add to corporate profits, all without taking up any shelf space.

MAM 2.0 supports the Long Tail. It leverages all assets, regardless of popularity or media type. It does this implicitly by collecting usage data and by using algorithms that mine these data for relevant patterns. It presents new displays and navigation paths to end users, paths that dynamically and continuously evolve as the data changes.

\section{Rich user experiences}

Historically, the web interface for enterprise MAM and content management systems was provided as an afterthought. Some companies built feature-limited web interfaces to the MAM. Other web interfaces were feature-rich, but extremely clunky. Some offered portal-style views into pre-arranged collections of assets and metadata. Finally, some simply publish a folder of assets to a web template. Most of these systems require a heavy amount of asset management on thick clients. Under that model, the web simply becomes another static presentation tool.

Rich User Experiences: Going beyond traditional web-page metaphors to deliver rich user experiences combining the best of desktop and online software. ${ }^{14}$

With MAM 2.0, the web interface provides a rich and rewarding user experience. Static presentation-only interfaces will not work. Even though the interface is completely web-based, it must have everything the user needs from their client solution. In effect, it should also eliminate their need for a client-side or a "MAM-only" plug-in. In order to do this, MAM 2.0 uses the latest web technologies, such as Ajax and Flash. More importantly however, MAM 2.0 systems are designed for the web, from the very beginning.
Rich user experience via 1-Click Launch ${ }^{T M}$ and web-based nonlinear editing

Web-based editing of documents, spreadsheets and video projects, asset annotation, streaming proxy videos, panning, zooming, and comparing images side-by-side, 1-click Launch from the web into production tools are all great examples of the rich user experience that a MAM $2.0 \mathrm{can}$ provide.

1-Click Launch, a process created by VFinity's founder Shen Tong, lets users take full advantage of their existing desktop tools, by providing a direct linkage between media, production tools, and the MAM all via the web. Effectively, 1-Click Launch is a way to open assets or projects directly into the appropriate production tool. It is not a client side plug-in; it is a completely web-based tool. 1-Click Launch is used to open video projects or Photoshop documents directly into Nonlinear Editors such as Final Cut Pro or image editors such as Adobe Photoshop.

Web-based Nonlinear Editing (Web-NLE) is another example of a rich user experience. Web-NLE is not something that popular video presentation systems such as YouTube are capable of supporting. While handling some aspects of metadata tagging and ranking via the web is already available, the time is approaching when it will be possible to handle many video production tasks completely via the web. Ingesting video from any source file or device, logging, clip, editing, re-mixing, transcoding and publishing video assets all via the web are functional areas that MAM 2.0 covers.

\section{Keep it simple, stupid}

Despite all the possibilities, it is important that Web 2.0 interfaces not be too complex. Complexity leads to low user adoption rates. Low user adoption rates lead to wasted money. If a system is too complex to use, then there is a great chance it will not be used.

Unfortunately, many early C/S solutions left us with a legacy, if not an expectation, that interfaces must have more features than are possibly needed. Divergent client requests resulted in numerous features that rarely got used by the broad base of end users. This "bloat" is an obstacle to user adoption.

The managed web experience is calculated simplicity. It offers only what the user needs, 
where and when then they need it. It adapts as user needs change. It should not force the user to change how they operate. It reduces, if not eliminates, the need for training. It dramatically increases user adoption of the solution. Ideally, it would even make the system fun to use.

\section{THE NEW ERA OF THE MANAGED WEB DEMANDS NEW DEVELOPMENT STRATEGIES}

All of this may sound fantastic, and it is, however, the real question is: how would it be possible to build a MAM 2.0 system and what will it cost? The great news is that a convergence of web-friendly production tools, advances in web technology, availability and bandwidth, place us at the beginning of a new era in digital content usage. For MAM 2.0, this means integrating only the most useful functionality of legacy solutions, but in a completely web-based and networked way. How to do this is a question for which the Web 2.0 principles "Perpetual Beta" and "Lightweight Models" provide answers.

\section{Continuous releases to meet ever- evolving needs: The Perpetual Beta}

Perpetual Beta: Move away from old models of software development and adoption in favor of online, continuously updated, software as a service (SaaS) models. $^{15}$

The traditional software release and adoption cycle more or less consists of the following phases: requirements analysis, design, implementation, testing, integration and maintenance. The timeline for most vendors is to devote a month or two to analysis. More time is devoted to design. Implementation takes four to five months. Quality assurance needs to be trained and testing will then begin with the first alpha, and so on. The end result is that the initial release cycle may take upwards of 12-18 months if everything goes right. If anything goes wrong along the way, such as a serious mismatch between the business requirement and technology delivery, then the process will need to be restarted. Traditional software development is a lengthy and costly process, and not well suited for MAM 2.0.

Developers of MAM 2.0 follow agile approaches. They release products early and often to meet dynamic business requirements. This is a dramatic change from legacy MAM which has many benefits. The entire idea-tocustomer cycle is much shorter. When the proper framework is in place, features are rolled out to customers in one to three month cycles. As a result, customer feedback may be received one month from the original idea, versus the six to twelve months in the old development model. Immediate feedback, based on actual usage, drives new functionality. This helps customers to understand how the system really impacts business processes. This is one of the major areas where a lack of understanding causes many enterprise implementations to fail. ${ }^{16}$

\section{Service-based and zero-footprint software}

Enterprise MAM is expensive. In MAM 2.0, expenses are reduced by implementing either zero-footprint software or software that is capable of being provided as a service. Below is a quick look at a cost comparison between DAM 1.0 and MAM 2.0 solutions (Figure 3). ${ }^{17}$

\section{Meet ever-evolving needs via Lightweight Models}

Lightweight Models and Cost-Effective Scalability: Use lightweight business- and software-development models to build products and business quickly and cost-effectively. ${ }^{18}$

Favoring simplicity is the best approach to meeting the needs of your end users. With MAM 2.0 the focus is on solving wellunderstood and real business requirements, not on perceived needs or rushing to build "metoo" software. Adhering to Web 2.0 principles will mean developing the minimum functionality, and designing the system to integrate with other business and open source systems to enable further collaboration.

MAM 2.0 provides opportunities to realize value from the existing managed web platform 
ENTERPRISE CLASS DAM

\begin{tabular}{|c|c|c|c|c|}
\hline $\begin{array}{l}\text { User Base: } 250 \text { creative users and } 5000 \text { consumer } \\
\text { users distributed globally }\end{array}$ & \multicolumn{2}{|c|}{ DAM Software - Internal Deployment } & \multicolumn{2}{|c|}{ Outsourced DAM Services Solution } \\
\hline STARTUP & YEAR 1 & 7-month deployment & YEAR 1 & 3-month deployment \\
\hline Applications and Software subtotal & $\$ 404,500$ & & $\$ 20,000$ & \\
\hline Labor and Consulting subtotal & $\$ 697,600$ & & $\$ 204,800$ & \\
\hline Hardware and Network Infrastructure subtotal & $\$ 328,000$ & & $\$ 0$ & \\
\hline TOTAL STARTUP COSTS & $\$ 1,430,000$ & & $\$ 224,800$ & \\
\hline OPERATIONS & YEAR 1 & 3-year total & YEAR 1 & 3-year total \\
\hline Applications and Software subtotal & $\$ 25,914$ & $\$ 199,314$ & $\$ 144,000$ & $\$ 528,000$ \\
\hline Labor and Consulting subtotal & $\$ 570,066$ & $\$ 3,284,666$ & $\$ 151,043$ & $\$ 812,543$ \\
\hline Hardware and Network Infrastructure subtotal & $\$ 73,080$ & $\$ 421,080$ & $\$ 0$ & $\$ 0$ \\
\hline TOTAL OPERATIONS COSTS & $\$ 669,060$ & $\$ 3,905,060$ & $\$ 295,043$ & $\$ 1,340,543$ \\
\hline TOTAL STARTUP AND 3-YEAR OPERATIONAL & & $\$ 5,335,160$ & & $\$ 1,565,343$ \\
\hline
\end{tabular}

EnterpriseDAM.1.0 ๑2006 GISTICS

Figure 3: Comparing costs of internally deployed DAM versus the on-demand DAM

in many ways. Functions such as search and retrieval are modularized and exposed as services. For example, this is used to expose assets to business-to-business portals. In MAM 2.0 , the work is performed quickly, not over the course of multi-year projects.

Innovation in Assembly: Building platforms to foster innovation in assembly, where remixing of data and services creates new opportunities and markets. ${ }^{19}$

VFinity receives numerous requests that we find are best managed following an "Innovation in Assembly" strategy. Some educational customers wish to integrate our MAM with open source image display and comparison systems. We are able to do so easily. Others wish to re-mix, reassemble and display existing assets in completely different ways. For example, they wish to assemble a source text-based asset in different ways to different audiences, including or excluding content, and changing styles as needed. All this and much more is possible with MAM 2.0 or managed web.

\section{What social software means to MAM: rights management}

While Web 2.0 principles are a clear benefit to enterprise MAM, one needs look no further than YouTube to see how the vast array of community contributed content causes headaches for content owners and distributors. With truly open enterprise MAM, users have unfettered access to production tools, and content sources. If they so choose, community members may rip video directly from digital video sources - the rights to which they do not own - mash these together, add unlicensed music, and post to YouTube instantaneously. Does this sound like a headache for Google? Well, it is.

As entertaining as the audience finds the material to be, significant rights violations are occurring with many submissions. The responsibility will be on the MAM 2.0 system to help manage all the myriad rights to all this content. While this seems like an impossible task, there are a number of strategies to make it manageable.

Managed web systems will need to integrate with web-friendly rights management solutions to start. The solutions can assist the MAM in scanning contributed content for any associated content rights. If violations are found, the assets can be moved to a special queue for further action. If rights are available, but need to be purchased, the MAM can integrate with payment solutions as necessary.

At the same time, the managed web can enlist its user communities to police themselves. For example, the community can flag assets for potential violations. These assets can automatically be moved to a special administrator's queue as needed. The same mechanisms that harness collective intelligence can be used as one part of the solution for managing digital rights. The managed web satisfies the needs of both the rights holders and the content consumers. While it is probably 
impossible to keep all objectionable material off the managed web at all times, it is a relatively simple task to combine the best of selfmanagement and systems-management to ensure that risks and violations are mitigated.

\section{Examples of what MAM 2.0 provides: Production from anywhere, any device, any tool}

What all of this provides is a significant increase in MAM richness and user involvement. The following case studies show a few real examples of what can be made possible with MAM 2.0.

\section{News team of one case study}

MAM 2.0 will result in more effective and efficient production for news operations. In today's worldwide news organizations content is typically gathered by a reporter and cameraperson. The material is either edited locally then fed to the broadcast center, or rough cuts can be fed in for final editing. Frequently, archival footage and/or graphics are inserted at the broadcast center before air. At that point the raw material will be sent to a central archive or stored in a bureau or production facility. The material often gets lost in the system because it is mis-logged or misplaced. Finding and acquiring the material involves valuable manpower.

Now fast forward to the MAM 2.0 news operation. A "news team of one" covers a story and returns to the satellite truck or bureau with their camera and raw material. They begin editing their own story on a laptop and through a web browser they are able to access their tools and search their news department's servers for additional information, video or graphics. The final piece is then sent to producers in the broadcast center and inserted in the program lineup. The raw material, including related research, is stored for future use as a file on a company server with uniform metadata and the reporter's comments.

\section{Virtual national focus group case study} Television networks spend billions of dollars each season on programs that they hope will attract a large number viewers that will result in substantial advertising revenue. With so much at stake, these companies invest a lot in research to help make smart programming decisions. This includes screening pilots for focus groups in Las Vegas and capturing their comments in a very manual cost-prohibitive process.

The MAM 2.0 research department will be able to virtually gather a focus group from anywhere in the nation and give them access to pilots right from their homes. A group of 2,000 can view a pilot and provide comments directly into web-based metadata fields. In turn, this input is directly available to programming executives. The information can also be analyzed by demographic and location. It will help producers, marketers and programmers to better understand the audience. And the information is stored with the program and easily accessible in MAM 2.0 at any future time. While bandwidth and security might be an issue today, soon technology will solve these issues.

\section{Content repurposing for publishers}

With declining subscription bases and advertising revenue, and the proliferation of freely available content on the web, media publishers are being forced to find new ways to generate revenue without increasing their workforce. One way to do this is to repurpose and resell old content. For any publisher without a managed web strategy this would be challenging. These publishers are forced to manually recreate new content from old, which comes at great expense.

Web 2.0 publishers find new value from existing content by repurposing what they already have. For example, they are converting text assets into new products, based on the needs of the intended audience. The same source content can be used to create multiple stylized products, each serving a unique need. The MAM performs all the processing, including or excluding content as needed, and wrapping it up into the desired publishing template for final presentation.

\section{Global story creation case study}

A good way to hold onto and/or grow an audience is to keep producing original and compelling content. The typical content creation process goes something like this: writer creates story; writer submits story; copy editor edits story; designer creates imagery; designer submits imagery; desktop editor creates page; copy editor proofs page; desktop editor makes final edits and 
changes; story is prepared for transmission; story is transmitted to printer; final product is archived. At every stage of the process, time is wasted searching for files, writing instructions, and distributing both to the next actor in the process. Each user may be working on disconnected devices and incompatible platforms. In the nonmanaged web space, most of the value is realized only in the final stage when the final product is archived.

MAM 2.0 publishers introduce efficiencies and value at every stage of the content creation process. The writer can create and submit the story from anywhere in the world. In fact, the writer can submit content directly into the MAM. The graphic designer can reside locally or remotely, and ingest their materials from any device. The copy editor can likewise make their edits from anywhere, work directly on the source material, and send change instructions immediately to the writer. The desktop editor is notified of the available project, searches the MAM for relevant materials, and saves significant time by re-using existing templates. The editor then launches their project from the managed web directly into desktop production tools. All work is routed and proofed via the MAM 2.0. Final versions are transmitted to the printing service, and the project is archived.

\section{SUMMARY}

Web 2.0 embodies a new era for the management and use of digital media. It has drastically increased the relevance of the online experience to producers and consumers. It has created a need to properly manage media across all devices and platforms. These events have created limitless opportunities to create new revenue streams from all assets, including seemingly less popular assets. It has also ignited a cataclysmic shift in traditional asset management strategies. In response, MAM 2.0 offers new solutions for how content creators and consumers can collaborate, and how the system can evolve independently as the needs of the user community change.

VFinity calls this reaction to Web 2.0, the "managed web" or "MAM 2.0". Under MAM 2.0, open, collaborative models connect media, metadata, end users and production tools via the web in fully networked and user-driven ways. Digital assets and projects are opened directly.
Any contribution to MAM 2.0 system is potentially a valuable data point for other members of the community. The managed web harnesses this data, using it to improve the system for other users.

MAM 2.0 responds to the openness of Web 2.0 by offering strategies that satisfy the needs of rights holders and content consumers. It enables greater collaboration between entire communities of users; content producers and consumers will be able to learn from each other on a scale previously unimagined. MAM 2.0 responds to the diverse needs of a global community by adopting agile development processes and offering only the most essential functionality in completely web-native tools.

MAM 2.0 is the next generation of MAM, empowering a global end user community with unlimited fully web-based access to:

- Media and metadata

- Production tools that combining best of web-based and desktop tools

- Intrinsically connected production tools and media

- Social software that becomes more useful the more it is used

- Rich user experiences that span all devices and platforms

These five elements provide a better way to enhance your productivity via the web. It is next evolution of the web; it is the managed web (MAM 2.0); and it is here today.

\section{References and Notes}

1 Robert, A. 29th August, 2005; Flickr Fans to Yahoo: Flick Off!; Wired News; Wired.com.

2 Yahoo, Inc PR department.

3 Vfinity's founder Shen Tong coined the term "Context is King", during a presentation entitled "Media Context from Taxonomy, Community Tagging, and Workflow Integration", at the 2006 Henry Stewart DAM \& MOM Symposium in Los Angeles, CA. It means that a piece of content becomes increasingly valuable as we capture how it is used and what users think of it, or the context.

4 http://www.zakon.org/robert/Internet/timeline/.

5 ibid.

6 ibid.

7 US Census Bureau. (2007) Statistical abstract of the United States; internet access and. http://www. census.gov/prod/2006pubs/07statab/infocomm. pdf. 
8 http://www.google.com/google-d-s/tour1.html.

9 Musser, J. with O'Reilly, T. and the O'Reilly Radar Team (2007). Web 2.0 Principles and Best Practices; O’Reilly Radar; Sebastopol, CA, pp. 10-11.

10 See ref 9 above.

11 ibid.

12 ibid.

13 See ref 9 above.

14 ibid.

15 ibid.
16 von Hellens, L., Nielsen, S. and Beekhuyzen, J. (2005) Qualitative Case Studies on Implementation of Enterprise Wide Systems. Idea Group Inc (IGI), Melbourne, p. 238.

17 Michael, M. and Joel, W. (2006) Business Case for On-demand Delivery of Digital Asset Management. GISTICS Incorporated; Oakland, CA, p. 11; data summarized from Enterprise Class DAM table.

18 See ref 9 above.

19 ibid. 